\title{
Autour des Orientales: Victor Hugo, Ernest Fouinet et la poésie arabe archaïque
}

About the Orientales: Victor Hugo, Ernest Fouinet and ancient arabic poetry

$$
\text { : فيكتور هوغو، ارنست فويني والشعر العربي القديمالشرقياتحول }
$$

\section{Pierre Larcher}

\section{OpenEdition}

\section{Journals}

Édition électronique

URL : http://journals.openedition.org/beo/1304

DOI : 10.4000/beo.1304

ISSN : 2077-4079

\section{Éditeur}

Presses de l'Institut français du Proche-Orient

\section{Édition imprimée}

Date de publication : 30 avril 2014

Pagination : $99-123$

ISBN : 978-2-35159-403-2

ISSN : 0253-1623

\section{Référence électronique}

Pierre Larcher, "Autour des Orientales : Victor Hugo, Ernest Fouinet et la poésie arabe archaïque »,

Bulletin d'études orientales [En ligne], LXII | 2014, mis en ligne le 11 juin 2014, consulté le 20 avril 2019. URL : http://journals.openedition.org/beo/1304 ; DOI : 10.4000/beo.1304 


\section{Autour des Orientales : Victor Hugo, Ernest Fouinet et la poésie arabe archaïque ${ }^{1}$}

Pierre LARCHER

Université d'Aix-Marseille / IREMAM

Les Orientales (1829) de Victor Hugo (1802-1885) sont en quelque manière à la littérature française ce que le West-östlicher Divan (1819 pour la $1^{\text {re }}$ édition, 1827 pour l'édition « élargie ») de Goethe (1749-1832) est à la littérature allemande : un chef d'œuvre de la littérature orientaliste, s'étant nourri de l'orientalisme savant et ayant inspiré à son tour la littérature ultérieure et au-delà... ${ }^{2}$

Le Divan est divisé en deux parties. La première comprend les poèmes, inspirés par la poésie persane et non pas arabe, la deuxième « des notes et dissertations pour une meilleure intelligence du divan occidendal-oriental ». La seconde de la soixantaine de notices en quoi consiste cette partie est intitulée Araber. Elle traite de la seule poésie préislamique, ne nommant que les Mu'allaqāt et leurs auteurs, d'après William Jones (1746-1794). Mais elle illustre cette poésie par le célèbre « chant de vengeance » de Ta’abbața Šarran, sans toutefois nommer ni le poète, ni sa source (Goethe, 1999, p. 268-275 ; cf. aussi Larcher 2012).

De manière comparable, mais non identique, et avec une ampleur bien moindre, les Orientales comprennent 41 poèmes, suivis de « Notes » renvoyant à 16 de ces poèmes. La note XI est la plus longue. Elle renvoie au poème XXVII, intitulé « Nourmahal la rousse ». Après avoir rappelé l'étymologie arabe de ce nom ( « lumière de la maison ») ${ }^{3}$, Hugo ajoute (1829, p. 399) :

«Bien que cette pièce ne soit empruntée à aucun texte oriental, nous croyons que c'est ici le lieu de citer quelques extraits absolument inédits de poèmes orientaux qui nous paraissent à un haut degré remarquables et curieux. La lecture de ces citations accoutumera peut-être le lecteur avec ce qu'il peut y avoir d'étrange dans quelques-unes des pièces qui composent ce volume. Nous devons la communication de ces fragmens, publiés ici pour la première fois, à

1 Version écrite de la conférence faite le 7 Juin 2012 à l'Université d'Aix-Marseille dans le cadre du séminaire du Centre interdisciplinaire d'étude des littératures d'Aix-Marseille (CIELAM EA4235) « Passages de frontières ». Je remercie André Miquel et Stéphane Baquey, ainsi que les deux rapporteurs anonymes, pour leur lecture et leurs commentaires. Dans les citations d'auteurs occidentaux, je conserve l'orthographe originale.

2 On ne résiste pas ici au plaisir d'une flèche : Edward Saïd (1935-2003), grand contempteur de l'orientalisme, tant savant que littéraire, n'en a pas moins donné, par une amusante palinodie, le nom de l'œuvre de Goethe à l'orchestre qu'il a fondé avec le chef Daniel Barenboïm...

3 Plus exactement «lumière du campement » (nūr al-mahall), mahall désignant étymologiquement l'endroit où on défait les bagages, et, par métonymie, celui où l'on s'installe. 
un jeune écrivain de savoir et d'imagination, Monsieur Ernest Fouinet, qui peut mettre une érudition d'orientaliste au service de son talent de poète. Nous conservons scrupuleusement sa traduction ; elle est littérale et par conséquent, selon nous, excellente ».

Ernest Fouinet (1799-1845) n'est plus aujourd'hui connu (et, encore, des seuls spécialistes!) que pour cette collaboration avec Hugo. Je ne suis évidemment pas le premier à m'intéresser au sujet. Assez récemment encore, Louca (2001, p. 29-31, repris, avec quelques remaniements, en 2006, p. 85-86), dans un alinéa intitulé « Arabisants pour deux géants ", met en parallèle le rôle de Fouinet auprès de Hugo avec celui de Kosegarten [i.e. Johann Gottfried Ludwig Kosegarten (1792-1860)] auprès de Goethe :

«Tel est aussi le cas d'Ernest Fouinet avec Victor Hugo. N'importe si, diplômé des Langues Orientales, Fouinet travaille au ministère des Finances. Les deux Parisiens se rencontrent autour de Charles Nodier, à l'Arsenal, centre effervescent du mouvement romantique. Hugo, qui compose alors Les Orientales, sans avoir fait d'études spécialisées, puise aux traductions dont le pourvoit l'enthousiaste Fouinet. En appendice à son recueil, il publie les plus extravagantes de ces traductions, comme un défi jeté aux théories du classicisme ».

Louca, cependant, tout Égyptien et arabophone qu'il était, ne s'intéresse ni à l'original arabe de ces extraits, ni à leur traduction, ni même à leur traducteur.

Un demi-siècle auparavant, Schwab, dans l'ouvrage (1950) tiré de sa thèse (1948), consacrait une notice (p. 351-353) à Fouinet, au Livre IV (« Le personnel »), chapitre III («Fondateurs et intermédiaires»), où on relève cette phrase (p. 352): «sous l'autorité de ce nom [celui de Hugo], il avait lancé dans la grande circulation littéraire la poésie antéislamique, les Orientales jouant ainsi en France un rôle symétrique à celui qu'avait le Divan en Allemagne ", ajoutant: "Grâce aux papiers de Fouinet envoyés tout droit par Hugo à l'imprimerie et adjoints aux notes de son recueil, ces grands noms se répandent: Imroulkaïs, puis Djelal-eddin-Roumi, Ferid-eddin-Attar, et Firdousi ». Cette formulation vient rappeler qu'à la suite des poèmes arabes, sont cités également des poèmes persans. En revanche, Hugo ne nomme pas Imroulkaïs...

Schwab se fonde pour l'essentiel sur un article de Martineau, paru en 1916 dans le Mercure de France et intitulé "Ernest Fouinet et les "Orientales" ", et dont il reproduit certaines erreurs, notamment une date de naissance fautive pour Fouinet (1790 au lieu de 1799) ${ }^{4}$. Mais Martineau a consulté le manuscrit des Orientales, conservé à la BnF et maintenant consultable sur le site Gallica, et constaté qu'il contenait un certain nombre de lettres de Fouinet (six, selon Martineau) d'où sortent non seulement les citations de la note XI, mais encore une bonne partie des commentaires.

L'édition dite critique des Orientales (Barineau, 1952-1954) ne mentionne qu'incidemment Fouinet dans l'Introduction (1952, p. IX-XXXIII), dénombrant dix lettres de Fouinet, un peu plus longuement dans la Notice sur Nourmahal la Rousse (1954, p. 78-79), d'où l'on détachera cette phrase: «Quelques critiques ont pensé que ces emprunts impliquent en-

4 On peut consulter la notice bio-bibliographique du Grand Dictionnaire universel du XIX ${ }^{e}$ siècle de Pierre Larousse (t. VIII, p. 658, 1872). 
vers Fouinet une grande dette non avouée et ont reproché à Victor Hugo d'avoir traité trop cavalièrement son ami ».

Ces " quelques critiques », non cités, peuvent être une allusion à un article de Pertuis, paru en 1924 dans le Mercure de France, où l'on peut lire (p. 411) : « Mais Victor Hugo n'accuse qu'une partie de sa dette à l'égard de Fouinet ; les traductions que l'orientaliste adressait au poète étaient accompagnées de commentaires que V. Hugo s'est appropriés sans plus de façon ».

Dans l'édition, considérée comme de référence, des œuvres complètes de Hugo (sous la direction de Jean Massin, Club Français du Livre, 1967), les Orientales sont présentées par le poéticien Henri Meschonnic (1932-2009). Ceci explique cela : qu'il soit aussi disert sur l'orientalisme littéraire de Hugo qu'économe sur sa connexion avec l'orientalisme savant, même si on y relève cette phrase (p. 486) : « Ses Notes et les extraits de traduction, inédites alors, de la poésie arabe montrent que sa documentation est réelle; c'est un initiateur autant qu'un suiveur ». Mais Fouinet n'est même pas nommé et les Notes données, non à la suite, mais directement sous les poèmes, sans autre forme de commentaire.

L'édition la plus récente des Orientales, celle de Laurent (Hugo 2000), n'ajoute très exactement rien sur le sujet. En fait, c'est dans une autre édition de référence des œuvres de Hugo, celle, très antérieure, d'Albouy, que j'ai trouvé mention d'une source de Fouinet, et, corrélativement, d'une appréciation de ses traductions (Hugo 1964, p. 1327 ; repris dans Hugo 1966, p. 368).

Stéphane Baquey (communication personnelle) m'assure qu'il manque une note savante d'un arabisant sur le sujet: la remarque d'Albouy, sur laquelle je reviendrai plus loin, ne présuppose pas, à la réflexion, qu'un arabisant soit déjà intervenu. C'est cette note qu'on lira ici en espérant ne pas répéter ce que d'autres avant moi auraient déjà dit.

\section{Les extraits}

Dans cette section, je «liste » les extraits de poèmes arabes. Si l'auteur est cité, je donne l'orthographe arabe exacte de son nom, en transcription, et identifie la pièce ; s'il ne l'est pas, je l'identifie, ainsi que sa pièce. J'ai collationné l'édition originale des Orientales (plus exactement, sous l'appellation de « cinquième édition », l'un de ses retirages) avec le manuscrit des Orientales, l'une comme l'autre disponibles sur le site Gallica de la BnF : le manuscrit des Orientales, auquel on renvoie par le numéro de folio, donne quelques indications absentes de la version imprimée. Voici le résultat:

1) «La chamelle», 13 vers, auteur cité

Ce premier extrait est conclu par son identification: «Ce morceau fait partie de la Moallakat de Tarafa » (Fouinet, 83r: Moallakat de Tarapha). Le commentaire qui suit est repris mot pour mot de Fouinet (83r) : «Tous les sept ans, avant l'islamisme.... Moallakat veut dire suspendue »: je le citerai in extenso dans la section 4. Le vers 1 est un ajout du traducteur destiné à situer le décor. Seul le toponyme Thamed figure dans le vers 1 de Ṭarafa, qui est: li-Hawlata aṭlālun bi-burqati Țahmadi /talūḥu ka-bāqū l-wašmi fì zāhiri l-yadi 
«De Khawla, les vestiges, à Thahmad, sont visibles / Tel reste de tatouage au revers de la main ». Mais l'examen du manuscrit montre que la lettre ${ }^{5}$ de Fouinet s'achève avant la citation (bas de 82r) par «mais la chamelle s'avance dans les sables de Thamed ", repris, à la page suivante (haut de $82 \mathrm{v}$ ) sous la forme «La chamelle s'avance dans les sables de Thamed ». Pour le reste, on reconnaît sans peine ici les vers 12, 14, 16, 17, 18, 19, 20, 22, 27, 29, 30 et 23 du texte donné par Zawzanī, m. 486/1093 (Šarh, p. 143-150). La question qui se pose ici est celle du déplacement d'un vers et de savoir s'il est le fait du traducteur ou de la version qu'il a suivie. C'est le second extrait qui permet en fait l'identification de sa source.

\section{2) « La cavale », 17 vers, auteur non cité}

Cet extrait n'est pas identifié. Mais Victor Hugo indique : " "Que les lecteurs d'un esprit prompt exercent sur ce tableau les forces de leur imagination" s'écrie, à propos de ce beau et bizarre passage, ce bon Allemand Reiske, qui préférait si énergiquement le chameau frugal de Tarafa au cheval Pégase » (c'est Hugo qui souligne). L'Allemand Reiske est Johann Jakob Reiske (1716-1774). On lui doit la première édition et traduction publiée d'une Mu'allaqa, précisément celle de Țarafa (orthographié Tharapha par Reiske et Tarapha par Fouinet), à Leiden, aux Pays-Bas, en 1742, avec les commentaires d'Ibn al-Naḥhās (m. 338/950). Si l'on se reporte à cet ouvrage, on vérifiera que, pour « La Chamelle », Fouinet donne les vers 12, 13, 15, 16, 17, 18, 19, 26, 29, 30 et 22 de cette version : c'est bien Fouinet qui déplace 22 pour en faire une conclusion, comme le prouve l'adjonction de «Enfin » («Enfin, elle ressemble à un aqueduc, dont le constructeur grec a couvert de tuiles le sommet »). Et, toujours si l'on se reporte à cet ouvrage, on tombe sur Pégase, dès la page IV du Prologue, et page V sur Tarafae frugi camelus. Dans les Notae, au vers 38, on trouve (p. 84-85) 18 vers d'une description d'un cheval, traduits en latin et attribués à Amralkeis [i.e. Imru' al-Qays], qualifié d' "Anacréon d'Arabie ». Ce sont bien ceux que l'on retrouve ici, à une exception près ( $\left.n^{\circ} 12\right)$. William Mac Guckin de Slane (1801-1878) donne p. 42-44 de son editio princeps du dīwān d'Imru' al-Qays (1837) une pièce de 43 vers où on reconnaît ces 18 vers (26-43), dans un ordre parfois différent. Wilhelm Ahlwardt (1828-1909), dans son édition (1870 [1913]) des «Six poésies », collection de six dīwāns, dont celui d'Imru' al-Qays, donne sous le $\mathrm{n}^{\circ} 19$ (p. 126-128) la même pièce, mais avec 42 vers, où on retrouve les mêmes vers (25-42) dans le même ordre. Si maintenant on se reporte au manuscrit, on voit que Fouinet envoie « La cavale » à Hugo dans une lettre du 2 Juillet [1828] ${ }^{6}$, où on lit (84r) :

«Si j'avais eu le plaisir de vous trouver lundi, j'aurais ajouté au manteau rayé que vous avez si bien ajusté à sa taille [i.e. celle de Mazeppa, cf. infra 7], une noble et puissante description

$5 \quad 82 \mathrm{r}, 82 \mathrm{v}$ et $83 \mathrm{r}$ constituent bien une seule et même lettre (lettre $\mathrm{n}^{\circ} 1$ de Martineau, qui l'a lue et transcrite), commençant par « Monsieur et ami » et se terminant par « Mille amitiés ». 83v est la suscription d'une lettre, écrite sur le pli extérieur et datée de Juillet 1828. Je ne puis dire si c'est celle de la lettre qui précède ou de celle qui suit, mais en tout cas, elle signale bien la frontière des deux lettres !

$684 \mathrm{r}, 84 \mathrm{v}$ et $85 \mathrm{r}$ constituent bien une seule et même lettre (c'est la deuxième lettre de Martineau, qui l'a lue et transcrite) commençant par « Monsieur et cher ami » et se terminant par « mes amitiés ». 85v est la suscription d'une lettre. 
de la cavale d'Amralkeis, poète arabe célèbre et contemporain de Mahomet ${ }^{7}$ : je vais vous le transcrire ici : elle pourrait éveiller en vous d'autres traits à ajouter à ceux dont vous avez ennobli le coursier sauvage de Mazeppa».

Suit la citation, après quoi Fouinet ajoute $(85 \mathrm{r}):$ « Que les lecteurs d'un esprit prompt exercent sur ce tableau les forces de leur imagination - dit Reiske après avoir fait connaître ce magnifique passage : cela va droit à vous et je crois que vous admirerez ». Chez Hugo, le vers 5 se termine par des points de suspension, assortis d'une note $\left(n^{\circ} 2\right):$ : il y a ici quelque chose de primitif et qui pourrait tout au plus se traduire en latin ». Je reviendrai sur ce point en 5 .

3) « Traversée du désert pendant la nuit » (3 vers) et

4) « Pendant le jour » (8 vers), auteur cité.

A la suite de ces deux extraits, Hugo indique : « ces deux tableaux sont d'Omaïah ben Aiëdz poëte de la tribu poétique des Hudeilites qui habitait au couchant de la Mecque ». Si on se reporte au manuscrit des Orientales, on voit qu'ils sont envoyés par Fouinet à Hugo dans une troisième lettre ${ }^{8}$, qui consiste pour l'essentiel en une traduction d'un long passage de Reiske (1742) sur les mérites comparés de Pégase et de la chamelle de Țarafa (Reiske préférant celle-ci à celui-là) que Fouinet conclut en ces termes (87r) :

«La diatribe est forte de la part d'un docte allemand, tout farci d'études classiques, mais je ne veux pas vous laisser de blanc et je vais vous conduire dans le désert sur un bon chameau, tel que Reiske les aime ; je serais heureux si le fragment que je vous donne pouvait être de quelque utilité à la composition de votre recueil d'Orientales ».

Suivent alors, non pas un, mais deux fragments, Fouinet concluant sa citation en ces termes (87v) : «Ce sont deux tableaux [Hugo a barré ce sont et remplacé par ces], qui pourraient vous être agréables [illisible] à [Hugo a barré tout cela et remplacé par d'] Omaiah ben Adjez, poète de la tribu poétique des Hudeilites qui habitait au couchant de la Mecque ». La jolie formule de Hugo est donc de Fouinet... Ce poète est facilement identifiable : il s'agit d'Umayya b. Abī al-'Á'id, tout à la fois poète de l'époque omeyyade (et de la tribu des Hudayl) : on peut renvoyer ici à Régis Blachère, Histoire de la littérature arabe, t. III, p. 601. Notons que le patronyme du poète (litt. « fils du père de celui qui se recommande [à Dieu] » suggère qu'il est de père inconnu. Les deux extraits de ce poète cités par Fouinet sortent eux aussi de Reiske (1742), qui le mentionne quatre fois dans ses Notae, sous des orthographes différentes: Ommia ben Abi Ajedz Hodeilitae, 1 vers, p. 48 ; Omaja ben Abi Ajeds, 8 vers, p. 87 ;Omaija ben Ajedz, 29 vers, p. 90-91; Omaja ben Abi Ajedz, p. 116, à nouveau pour le troisième des 8 vers de la p. 87 . Le patronyme raccourci de Fouinet vient de la troisième

$7 \quad$ En fait, Imru' al-Qays (fin $\mathrm{V}^{\mathrm{e}}$-début $\mathrm{VI}^{\mathrm{e}}$ siècle ap. JC) est très antérieur à Mahomet.

$8 \quad 86 \mathrm{r}, 86 \mathrm{v}-87 \mathrm{r}$ et $87 \mathrm{v}$ constituent bien une seule et même lettre (la troisième de Martineau, qui l'a lue et transcrite), commençant par « Monsieur et ami » et se terminant par « Votre ami, j'aime à le répéter ». On ne peut donc pas dire, avec Martineau, que « Suivent les traductions de deux poèmes, Traversée du désert pendant la nuit et pendant le jour ». Celles-ci sont en fait avant la formule finale. En revanche, Martineau a bien vu que «Pendant la nuit » n'était pas un titre autonome, mais dépendait de « Traversée du désert ». 
mention de Reiske. Les huit vers de «Pendant le jour » viennent également de cet extrait de 29 vers, dont Fouinet traduit les vers 18, 19, 20, 21, 23, 24, 25 et 29. En revanche, " pendant la nuit », vient du second extrait, de 8 vers, cité par Reiske et dont Fouinet traduit les cinq premiers (ses vers 2 et 3 joignent 2-3 et 4-5). Dans le dīwān des Hudayl, publié partiellement par Kosegarten en 1854 sous le titre de Carmina Hudsailitarum, la poésie de Umayya b. Abī al-' $\bar{A}$ 'i commence p. 176 et comprend les pièces suivantes : $n^{\circ} 90$ (29 vers, p. 176-179), $n^{\circ} 91$ (6 vers, p. 180), n 92 (83 vers, p. 180-198), nº 93 (51 vers, p. 198-203), nº 94 (6 vers, p. 203), $n^{\circ} 96$ (11 vers, p. 205-207), qui est une réponse à son oncle maternel Sahm b. Usāma ( $n^{\circ} 95$, 17 vers, p. 204-205), à laquelle répond à son tour son cousin Iyās b. Sahm b. Usāma (n 97, 41 vers, p. 207-210), nº 98 (23 vers, p. 210-212, réponse à la réponse du précédent), nº 99 (35 vers, p. 212-215), n 100 (27 vers, p. 215-217), soit 9 pièces. Le premier des deux extraits est constitué des vers 28-32 de la pièce $n^{\circ} 99$ (p. 214) ; le second, des vers 25-28, 37-39 et 43 de la pièce $n^{\circ} 93$ (p. 200-202).

5) Sans titre (début : « La fortune... »), 7 vers, auteur non cité

Ce cinquième extrait est introduit par «voici un fragment, plus ancien encore, admirable de profondeur et de mélancolie. C'est beau autrement que Job et Homère, mais c'est aussi beau ». Si on se reporte au manuscrit des Orientales, on voit qu'il est envoyé par Fouinet à Hugo dans une quatrième lettre ${ }^{9}$ commençant par ces mots (88r) : « Vous avez la bonté de penser à moi et à mes [illisible et omis par Martineau !] ; moi je pense à vous et à vos Orientaux. Je viens de lire un petit poème bien court, mais tout primitif. Je vous le fais connaître : "La fortune..." ", Fouinet concluant (88v) : «Voilà bien le cœur de l'homme et ses sentiments profonds. La nouvelle école [soulignée par Fouinet] est des premiers temps du monde » et ajoutant : « et ces deux vers » [extrait non repris par Hugo] :

«Je suis descendu vers les gens de Mahleb, dans l'hiver, étranger, sans asyle; c'était au temps de la disette ;

Je n'ai cessé d'éprouver leurs bienfaits ; ils m'ont couvert ; ils m'ont fait du bien, tellement que je me suis cru dans ma tribu».

Commentaire de Fouinet (89r) : «L'hospitalité est-elle bien peinte dans ces deux lignes simples? ${ }^{10}$. Fouinet ne donne aucune indication permettant d'identifier ces deux extraits, l'un repris et l'autre non. Hugo parle pour le premier d'un « fragment plus ancien encore », indication qui ne peut venir que d'une communication orale de Fouinet. Si par «plus ancien », il faut entendre plus ancien que les deux morceaux qui précèdent et qui sont d'un poète hudaylite d'époque omeyyade, on pourrait penser, les deux morceaux suivants étant aussi de poètes hudaylites, que ces deux extraits proviennent eux-mêmes du dīwān des Hudayl, le premier des deux plus spécialement d'un poète préislamique. Malgré

9 88r-88v-89r constituent bien une seule et même lettre, commençant par « Monsieur et cher ami » et se terminant par «Au lieu de coups, mettez amitiés et tout sera vrai ». $89 \mathrm{v}$ est la suscription.

10 Cette lettre est cependant très mal publiée par Martineau (1916). Il enchaîne directement «je vous le fais connaître... » et « voilà bien le cœur de l'homme... » qui, du coup, « ces deux vers » étant omis, ne commentent plus l'extrait $n^{\circ} 5$, mais introduisent l'extrait non repris par Hugo! 
l'anaphore de «La fortune... » (en espérant qu'elle imite l'arabe), malgré la présence des oiseaux qațā, qui ont donné leur nom au ganga (ganga alchata), malgré la présence du nom propre Mahleb dans l'extrait non repris, je ne les y ai pas trouvés : c'est mon seul échec...

6) « Rencontre de tribus », 4 vers, auteur non cité

Il n'y pas de commentaire chez Hugo. Si on se reporte au manuscrit des Orientales, on voit que cet extrait est envoyé dans une lettre, la cinquième ${ }^{11}$, à Hugo (avec en-tête, barré, Administration des contributions indirectes), où il est introduit en ces termes (90r) : «Voici d'abord quelques vers que composa Abd Manaf [mal lu par Martineau en Abdmonay], dans le temps d'ignorance, c'est-à-dire avant Mahomet. Ils renferment la description d'une rencontre de tribus. Le fragment est court, mais il est si poétique ! et il peut fournir de belles lignes à votre préface ». Le titre de l'extrait, on le voit, vient de Fouinet. Lequel conclut en ces termes $(90 \mathrm{v})$ : «Voilà bien de la poésie de tous les temps, de toutes les écoles, c'est celle de votre phalange où je m'engage comme lieutenant ». Hugo ne reprend ici aucun des commentaires de Fouinet. En revanche, il reprend ses notes (90r-90v) :

\begin{abstract}
«Le poète ne se serait point borné à dire un nuage dans ce cas : un nuage est bienfaisant pour les Arabes. Mais il dit un nuage plein de grêle, malfaisant » [Fouinet : Il n'aurait pas dit un nuage, le reste sans changement]

« La [Fouinet: Maudite] langue française [qui] n'a pas de mot pour rendre ce bruit de l'eau qui tombe dans l'eau: les Anglais ont une expression parfaite, splash. [phrase non reprise] Le mot arabe est bien imitatif aussi, ghachghacha » [Hugo a barré la suite : Prononcez le gh comme si vous gargarisiez, vous aurez une onomatopée parfaite].
\end{abstract}

Le seul 'Abd Manāf connu est l'arrière-arrière grand-père de Mahomet (fils de 'Abd Allāh, fils de 'Abd al-Mutțalib, fils de Hāšim, fils de 'Abd Manāf). Mais 'Abd Manāf est aussi le nom d'un de ses oncles paternels, plus connu sous le nom en forme de teknonyme (kunya) d'Abū Țālib, et auquel la tradition islamique attribue bien en effet des vers : on ne retrouve cependant l'extrait dans aucun des quatre poèmes cités par Ibn Hišām, m. 213/828 ou 218/833 (Sïra, I, p. 280-281, 282, 286-298, 393). J'ai espéré retrouver le poème par le mot

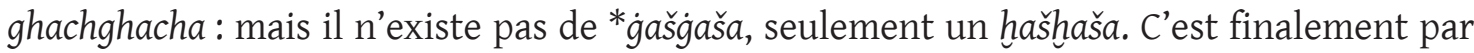
l'index de la Hizānat al-adab de Bağdādī (m. 1093/1682), au premier chef commentaire de « vers témoins » (šawāhid) d'un ouvrage de grammaire, mais en fait véritable encyclopédie littéraire, que j'ai trouvé trace et du poète et de son poème. Il s'agit d'un poète de la tribu des Hudayl : 'Abd Manāf b. Rib' al-Hudalī al-Ǧurabī. Bağdādī indique que le vers témoin ( $n^{\circ} 506$ ) est le dernier d'un poème de 12 vers qu'il cite (t. VII, p. 45-46) et dont Fouinet a traduit les vers 8, 9, 10 et 11. On trouve, au vers 9, le mot... šágšága (auquel Fouinet a fait subir une métathèse : il aurait dû écrire chaghchagha). On trouve également le texte arabe

11 90r-90v-91r-91v constituent bien une seule et même lettre (lue et transcrite par Martineau), commençant par " Je raye bien vite, monsieur et cher ami... » et se terminant par « Ernest. Abd Allah Hodeily ». Elle contient donc les extraits 6 (90r-90v) et $7(90 \mathrm{v}-91 \mathrm{r}-91 \mathrm{v})$. 
de ce poème dans la dernière partie du dīwān des Hudayl publiée par Julius Wellhausen (1844-1918) ${ }^{12}$, en 1884, p. 3 de la partie arabe, sous le nº 139.

7) Sans titre (« Tous les soirs... »), 11 vers, auteur cité

Cet extrait est introduit par Hugo en ces termes : "le morceau suivant qui est de Rabiah ben al Kouden nous semble remarquable par le désordre lyrique des idées. Il est curieux de voir de quelle façon les images s'engendrent une à une dans le cerveau du poète, et de retrouver Pindare sous la tente de l'Arabe ». Si maintenant on se reporte au manuscrit des Orientales, ce morceau suit le précédent, introduit par Fouinet en ces termes $(90 \mathrm{v})$ : «Passons à Rabiah ben al Kouden, il dit:... ». Suit la citation, mais que Fouinet entrecoupe de commentaires, après le vers 3 et après le vers 7 , suivis d'une conclusion : ni les commentaires, ni la conclusion ne sont repris par Hugo, mais nous y reviendrons dans les sections 4 et 5. Quant à l'auteur, il s'agit en fait de Rabía b. al-Kawdan, également poète de la tribu des Hudayl. Fouinet ne le dit pas explicitement, mais le sait, puisqu'il signe sa lettre Abd Allah Hodeily (autant dire le Hodeilite, Abd Allah «serviteur de Dieu » pouvant s'appliquer à tout individu croyant ) ! Le morceau de Fouinet ne vient pas de Reiske, qui cite toutefois ce poète sous la forme Rabia ben al-Kardan, p. 77, mais pour deux vers seulement. On peut trouver le texte arabe de ce poème dans les Carmina Hudsailitarum publiés par Kosegarten en 1854 ( $n^{\circ} 134$, p. 288-291). C'est un poème de 19 vers, dont Fouinet traduit 11 $(1,2,3$ puis $6,7,8,9$, puis $12,13,14,15)$.

\section{8-14) Extraits du Hamasa}

Après ces sept premiers extraits, Hugo indique : «Les extraits qu'on va lire sont du Hamasa, et sont inédits, en France du moins, car une édition de ce grand recueil s'imprime en Allemagne avec une version latine ». Un arabisant dirait, Hamasa étant du féminin, la Hamāsa : il y a deux Hamāsa, celle d'Abū Tammām (m. 231/845 ou 232/846) et celle d'alBuhturī. Un spécialiste identifiera sans peine la Hamāsa avec celle d'Abū Tammām pour trois raisons, dont les deuxième et troisième seront indiquées en leur lieu et place, mais dont la première se trouve dans le chapeau introductif. "L'édition de ce grand recueil qui s'imprime en Allemagne avec une version latine » est une allusion aux Hamasae Carmina de Georg Wilhelm Friedrich Freytag (1788-1861), dont le tome I (texte arabe) paraîtra à Bonn cette même année 1828, tandis que les tome II et III (traduction latine) paraîtront une vingtaine d'années plus tard en 1847 et 1851. Ces sept extraits sont dans l'ordre :

1) (extrait $n^{\circ} 8$ ) Sans titre, 4 vers, « Kotri ben al Fedjat el-Mazeni dit : »

2) (extrait $n^{\circ}$ 9) Sans titre, 4 vers, «Chemidher el Islami, du temps de l'Islam, dit (après avoir tué celui qui avait tué son frère par surprise) : »

12 Wellhausen est bien connu comme l'homme qui a fait prendre aux études bibliques le virage « philologique » (vs « théologique »). 
3) (extrait $n^{\circ}$ 10) Sans titre, 6 vers, « Du temps de l'Islam, Oueddak ben Tsomeïl dit: (La tribu de Mazen, dont faisait partie le poëte, possédait près de Barrah un puits nommé Safouan. Les Benou Scheiban le lui disputèrent. Tel est le sujet.)»

4) (extrait $\left.n^{\circ} 11\right)$ Sans titre, 6 vers, «Salma ben Iezid el Djofi, sur la mort d'un frère :» Sous l'appellation « Fragments »

5) (extrait $\left.n^{\circ} 12\right)$ Sans titre, 2 vers, (« auteur inconnu »)

6) (extrait $\left.n^{\circ} 13\right)$ Sans titre, 3 vers « Rita, fille d'Asem, dit : »

7) (extrait $\left.n^{\circ} 14\right)$ Sans titre, 2 vers, « Abd-eben-al-Tebib dit : »

«Ces quatre derniers morceaux [i.e. 11-14] sont tirés de la seconde partie du Hamasa: cette seconde partie a pour titre : Section des chants de mort». Ce commentaire confirme qu'il s'agit de la Hamāsa d'Abū Tammām : celle-ci est en effet divisée en un certain nombre de chapitres $(b \bar{a} b)$, dont le second porte le nom de bāb al-marātī ou « chapitre des thrènes ».

On note tout de suite la bizarrerie. Après avoir donné quatre premiers extraits, Hugo donne sous l'appellation « Fragments » trois autres extraits, mais en indiquant que les quatre derniers morceaux sont extraits de la deuxième partie de la Hamāsa. Il y a un chevauchement entre les deux séries. Si on les symbolise respectivement par (...) et [...], il peut être ainsi représenté : (1-2-3-[4)-5-6-7]. Si l'on se reporte au manuscrit des Orientales ${ }^{13}$, on constate que Fouinet (92v) donne : 1) Salma ben Iezid al-Djofi sur la mort d'un frère ; 2) Fragment (au singulier) (auteur inconnu) ; 3) Rita fille d'Asem dit ; 4) Abd-ebn al-Tebib dit ; 5) Motammen ben Nowaïs [sic], non repris ${ }^{14}$, indiquant (93r) : « Les chants qui précèdent sont tirés de la seconde section du recueil intitulé Hamasa : cette seconde section a pour titre Section des chants de mort ». Plus loin (98r-98v-99r), Fouinet donne : 1) un des Beni Temim a dit (non repris) ${ }^{15}$;2) Kotri ben al-Fedjat el-Mazeni ;3) Chimizer el-islami (du temps

13 92r-92v-93r-93v-94r-94v-95r-95v sont une autre lettre qui contient cinq extraits de la Hamāsa (92r-92v-93r), dont quatre repris par Hugo (extraits 11-14), suivis des deux extraits du dīwān des Hudayl (93r-93v-94r-94v-95r, extraits 15-16). On n'a ni le début, ni la fin de cette lettre. En 95v, on lit : «J'enveloppe de la poésie avec de la poésie. Le bureau m'a empêché de vous en traduire davantage. Je vous en promets plus pour une autre fois ». Martineau (1916) ignore ces feuillets.

14 Il s'agit en fait de Mutammim b. Nuwayra, dont 3 vers sont cités dans la Hamāsa (t. II, p. 148-149) et dont Fouinet traduit les deux premiers $(93 \mathrm{r}):$ : Il m'a blâmé quand il m'a vu près du tombeau versant des larmes abondantes. Il m'a dit : - est-ce que tu pleures sur chaque tombeau que tu vois ployé entre des monticules de sable?».

$1598 \mathrm{r}$ « Un des Beni Temim a dit :

J'ai vu la cavalerie, au moment où elle chargea, frapper à coups redoublés pour le loubabah (manteau de guerre) de Motamattir de Lakhm

Elle se précipitait sur eux comme se précipite la chamelle sur celui qui vient lui enlever son petit ».

Ce poème est suivi d'un habar, c'est à dire du récit des événements que le poème est censé illustrer : "Ces deux vers sont antérieurs à l'islamisme et furent composés après la journée d'Ouara, lieu célèbre par une cruauté commise sur les enfants de Darim par Amr ben Hind. Un de ceux-ci avait tué son fils, par accident; il jura de brûler cent beni Darim. Il n'en trouva que quatre-vingt dix-huit; il y ajouta un homme des Beradjem et une femme des Nahchals, d'où vient qu'il est surnommé le brûleur et, à cause de sa dureté, "qui écrase les pierres" ». Il y a bien « un des Beni Temim » (rağul min Banī Tamīm) dans la Hamāsa (t. I, p. 112), mais c'est un autre extrait de 4 vers. En revanche, dans l'environnement immédiat des trois autres extraits, on trouve « un des Ban̄̄ Taym Allāh b. Ta laba » (t. I, p. 66-68), extrait de trois vers dont Fouinet «traduit » en quelque manière 1 et 3. Taym a été confondu avec Tamīm. Il est suivi du récit de la journée de Uwāra, où l'on retrouve la première 
de l'islam) ; 4) Oueddak ben Tsomeïl el-Mazini, indiquant (99r) : «Ces poèmes sont du Hamasa et sont inédits en France du moins, car une édition s'imprime en Allemagne, avec une version latine ». Chez Fouinet, ces quatre extraits sont les derniers cités de poèmes arabes et sont suivis par des extraits de poèmes persans. Ils ont donc été déplacés pour être réunis avec les quatre extraits du livre II de la Hamāsa, eux-mêmes appartenant au livre I, appelé Hamāsa (comme l'ensemble du livre) ou livre de la bravoure ou courage à la guerre...

On peut résumer dans le tableau suivant les différences entre Hugo et Fouinet:

\begin{tabular}{|c|c|}
\hline Hugo & Fouinet \\
\hline 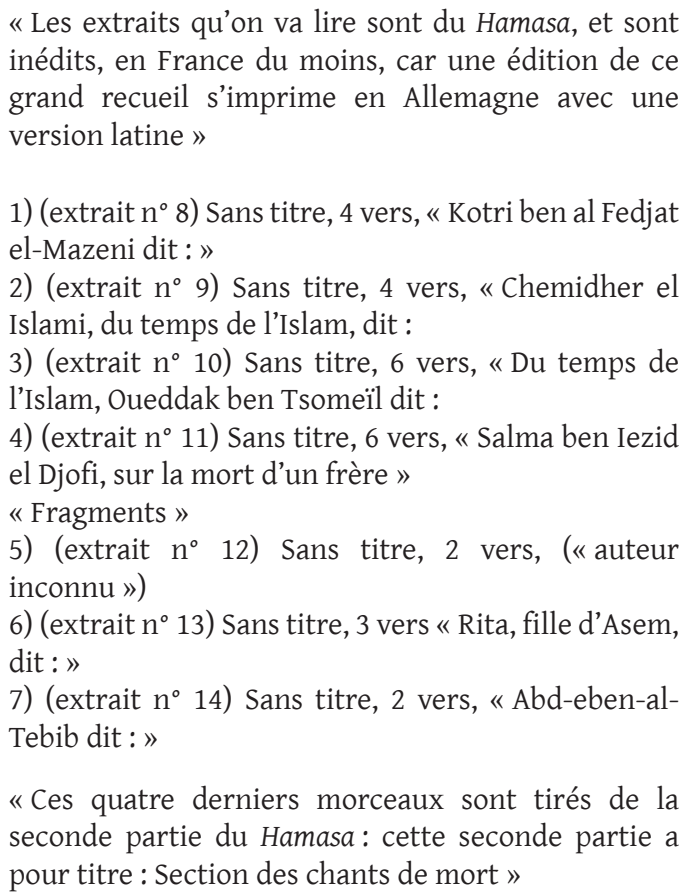 & $\begin{array}{l}\text { 1) (extrait } n^{\circ} \text { 11) Salma ben Iezid al-Djofi sur la mort } \\
\text { d'un frère } \\
\text { 2) (extrait } n^{\circ} \text { 12) Fragment (au singulier) (auteur } \\
\text { inconnu) } \\
\text { 3) (extrait } n^{\circ} \text { 13) Rita fille d'Asem dit } \\
\text { 4) (extrait } n^{\circ} \text { 14) Abd-ebn al-Tebib dit } \\
\text { 5) Motammen ben Nowaïs [sic], non repris } \\
\text { « Les chants qui précèdent sont tirés de la seconde } \\
\text { section du recueil intitulé Hamasa: cette seconde } \\
\text { section a pour titre Section des chants de mort» } \\
\text { 1) un des Beni Temim a dit (non repris) } \\
\text { 2) (extrait } n^{\circ} \text { 8) Kotri ben al-Fedjat el-Mazeni } \\
\text { 3) (extrait } n^{\circ} \text { 9) Chimizer el-islami (du temps de } \\
\text { l'islam) } \\
\text { 4) (extrait } n^{\circ} \text { 10) Oueddak ben Tsomeill el-Mazini } \\
\text { "Ces poèmes sont du Hamasa et sont inédits en } \\
\text { France du moins, car une édition s'imprime en } \\
\text { Allemagne, avec une version latine». }\end{array}$ \\
\hline
\end{tabular}

On présentera de même, sous forme d'un tableau, l'identification des poètes et de leurs pièces :

1) Kotri ben al Fedjat el-Mazeni

Il s'agit de Quțīì b. al-Fağăa al-Māzinī : il y a trois pièces de lui dans la Hamāsa : t. I, p. 49-50 (7 vers) ; t. I, p. 68-69 ( 4 vers) et t. II, p. 111 : ce sont les quatre vers du second extrait qui sont traduits dans les Orientales

2) Chemidher el Islami

Il s'agit de Šamaydar al-Hāritîi, dont une pièce est citée dans la Hamāsa, t. I, p. 61. Ce sont les quatre vers de l'extrait qui sont traduits

phrase de Fouinet, mais non la suite. On y trouve la variante libäba, avec la paraphrase « habit de guerre », au lieu de kināna (« carquois ») dans le texte et al-Mutamațțir avec l'interprétation possible « homme des Lahm ». Tout cela suggère que Fouinet et/ou sa source ont utilisé une version de la Hamāsa, avec un autre commentaire que celui de Tibrīzī (m. 502/1109). 


\begin{tabular}{|c|c|}
\hline 3) Oueddak ben Tsomeïl & $\begin{array}{l}\text { Il s'agit de Waddāk b. Tumayl al-Māzinī, dont deux } \\
\text { pièces sont citées dans la Hamāsa, t. I, p. } 63 \text { ( } 6 \text { vers) } \\
\text { et t. II, p. } 112 \text { ( } 3 \text { vers) : ce sont les six vers du premier } \\
\text { extrait qui sont traduits }\end{array}$ \\
\hline 4) Salma ben Iezid el Djofi & $\begin{array}{l}\text { Il s'agit de Salma al-Ǧu'fī, Hamāsa, t. III, p. 59-60, } 6 \\
\text { vers, ici traduits }\end{array}$ \\
\hline 5) auteur inconnu & $\begin{array}{l}\text { Ce extrait se trouve dans la Hamāsa, t. III, p. 64-65 et } \\
\text { comprend six vers, dont sont ici traduits les deux } \\
\text { premiers }\end{array}$ \\
\hline 6) Rita, fille d'Asem & $\begin{array}{l}\text { Il s'agit de Rịta bint 'Āṣim, Hamāsa, t. III, p. 69-70, } 5 \\
\text { vers : sont traduits les vers 2-3-4 }\end{array}$ \\
\hline 7) Abd-eben-al-Tebib & $\begin{array}{l}\text { Il s'agit en fait de 'Abda b. al-Ṭabīb, Hamāsa, t. II, } \\
\text { p. } 145-147,3 \text { vers: sont traduits les vers } 1 \text { et } 3 \text {. La } \\
\text { traduction du premier vers est incomplète, ne } \\
\text { rendant que 'alayka salāmu llāhi Qaysa bna 'Āsimin / } \\
\text { wa-rahmatuhu "Sur toi, le salut de Dieu, Qays ben } \\
\text { 'Âsim, / et sa miséricorde " mais non la fin mā šäa } \\
\text { llāhu an yatarahhamā, d'interprétation difficile. On } \\
\text { dit bien 'Abda, avec taskin (sans vocalisation du b) } \\
\text { mais 'Alqama b. 'Abada, avec tahrīk (vocalisation du } \\
\text { b) selon Lisān al-'Arab (art. 'BD, t. II, p. 666). Cf. églt } \\
\text { Blachère, Histoire de la Littérature arabe, t. II, p. } 259 \text {. }\end{array}$ \\
\hline
\end{tabular}

\section{5-16) divan de Hodeil}

«Les morceaux qui suivent sont tirés du divan de la tribu de Hodeil » (Fouinet, 93r: «les vers qui suivent sont du divan de Hodeil [deux mots illisibles] »). Il y a un premier morceau, suivi de quatre autres :

1) Sans titre, 7 vers, Barik

2) Fragments, 12 vers, auteur(s) non cité(s)

Le premier morceau est précédé d'un habar, entièrement repris de Fouinet (93r-93v) :

"Taabatà Cherrân (un des héros du désert) et deux de ses compagnons rencontrèrent Barik. Celui-ci s'éloigna d'eux, monta sur un rocher, ensuite il répandit ses flèches à terre. - Oh! l'un de vous, dit-il, sera mort le premier ; un autre le suivra ; et, quant au troisième, je le secouerai comme le vent fait de la poussière. "Et Barik fit là-dessus ces vers : C'était dans le pays [Fouinet : dans le lieu] de Thabit... . La fin de ce poème est un peu obscure, c'est le défaut de toute haute poésie, et surtout de toute poésie spéciale et primitive [Fouinet : le poème est un peu obscur : c'est une rencontre d'ennemis]. »

Après les quatre fragments, Hugo conclut: «Il y a encore de l'obscurité dans ces fragments, mais il nous semble que la grâce et le sublime percent au travers ». Ce commentaire ne vient pas de Fouinet qui se contente de répéter (95r) : « Extraits du divan de la tribu d'Hodeil ».

Ta'abbața Šarran, que l'on a déjà rencontré en introduction et que l'on retrouvera en conclusion, est le surnom d'un des deux plus célèbres des poètes-brigands de l'Anté-Islam, l'autre étant Šanfarā. Il est ici, sous son nom de Tāabit [b. Ǧābir], au centre d'un poème du 
poète hudaylite «Barik ». Mais Barik est une mauvaise lecture du ductus b-r-y-q : il s'agit en fait d'al-Burayq b. 'Iyāḍ b. Huwaylid al-Hunāì. On peut trouver le texte arabe du habar, suivi d'un poème de 16 vers, sous le $n^{\circ} 171$, et comme venant du seul al-Ǧumahị [i.e. Ibn Sallām al-Ǧumahī, m. 231/845 ou 232/846] dans Wellhausen (1884, p. 22-23 de la partie arabe), ainsi que leur traduction dans la partie allemande du même ouvrage (p. 129-130). Fouinet, quant à lui, traduit les vers 1,2, 3, 4, 5, 7 et 8 de ce poème.

Pour les quatre fragments qui suivent, aucun auteur n'est cité. Ayant cependant observé que Reiske (1742) faisait de très nombreuses citations du dīwān des Hudayl, j'en ai fait le relevé et ainsi découvert que le vers 1 du deuxième fragment se trouvait page 48 : ce vers est en fait la première des quatre citations que fait Reiske du poète hudaylite Umayya $b . A b \overline{1}$ al-'Á'id. Me reportant ensuite à Kosegarten (1854), je l'ai trouvé comme vers 9 de la pièce $n^{\circ}$ 96, mais j'ai également constaté que le vers 10 de cette même pièce était en fait le vers 2 du deuxième fragment de Hugo. Fouinet citant deux vers, sans donner le nom du poète, alors que Reiske n'en cite qu'un, en donnant le nom du poète, on peut raisonnablement conclure que Reiske n'est pas ici la source de Fouinet. La suite confirme cette hypothèse. Dans le quatrième fragment, de 7 vers, le toponyme Madjel, à la fin du vers 2, donc donnant la rime (en lām), m'a permis de retrouver ce vers comme le vers 2 de la pièce $n^{\circ} 95$ de Kosegarten (1854, p. 204). Cette pièce de 17 vers est de Sahm b. Usāma et Fouinet en traduit les vers 1-6, puis 8. Le « Omaya » du vers 2 est Umayya b. Abī al-'Á'id, dont Sahm était l'oncle maternel. C'est, dit le commentaire introductif, une composition érotique (yušabbib) sur une femme prénommée Laylā. C'est à cette pièce que répond Umayya dans la pièce suivante $\left(n^{\circ} 96\right)$, selon le commentaire introductif, et le premier des quatre fragments ( Tu as loué Leïla en rimes qui, par leur enchaînement, donne l'idée d'une étoffe rayée d'Yémen ») «traduit » en quelque manière le vers 1 de cette pièce : tamaddahta Laylā fa-mtadih Umma Nāfiin/ bi-qāfiyatin mițli l-habīiri l-musalsali «tu as loué Laylâ : loue donc Umm Nâfi' / D’une rime pareille à l'[habit] rayé qui s'enchaîne ". Quant au troisième fragment, ce sont les vers 5 et 6 de la pièce $n^{\circ}$ 97, du cousin d'Umayya, Iyās b. Sahm b. Usāma, répondant à sa pièce 96. Le fait que les quatre fragments appartiennent à des pièces qui non seulement se suivent, mais encore constituent un ensemble permet de faire une hypothèse quant à la source de Fouinet, comme nous le verrons bientôt.

17) Sans titre, 6 vers, auteur cité

«Voici le début du poëme de Chanfari [sic], poëte de la tribu d'Azed et coureur de profession », dit Hugo. On reconnaît sans peine ici le début (v. 1-7) de la lāmiyyat al-'Arab de Šanfarā (Chanfari provient du fait qu'on orthographie de la même manière $\bar{\imath}$ et $\bar{a}$ en finale, les points diacritiques qui les distinguent étant souvent omis). Le vers 5 de l'extrait condense cependant les vers 5 et 6 de l'original. Chanfari pour Šanfarā signale pratiquement

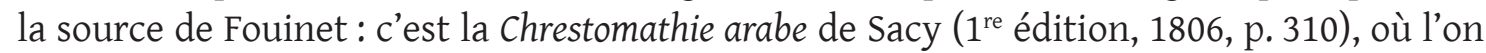
peut lire qașīdat al-Šanfarī al-mawsūma bi-lāmiyyat al-'Arab (en revanche Chanfara, dans la table des matières, p. 581). Erreur corrigée dans la seconde édition (1826, t. II, p. 345), Sacy signalant que l'erreur vient de d'Herbelot [i.e. Barthelémy d'Herbelot (1625-1695), l'auteur 
de la Bibliothèque orientale (1697)] et Pococke [i.e. Edward Pococke (1604-1691), l'auteur de Specimen Historiae Arabum (1650)]. Si l'on se reporte au manuscrit des Orientales, on voit que cet extrait est envoyé à Hugo par Fouinet dans une nouvelle lettre (la sixième, selon Martineau, mais on a vu que ce numéro d'ordre n'était pas adéquat) ${ }^{16}$, où, après avoir parlé d'autre chose, il écrit (96v) : « je vais vous fournir à présent le petit dessert oriental. C'est le début d'un poème composé par Schanfari poète de la tribu d'Azd et coureur de profession ». Le commentaire de Hugo qui clôt la citation est mot à mot celui de Fouinet (97r) : « Quel ton de grandeur, de tristesse et de fierté dans ce début! Tel est le caractère général de ces poëmes de cent vers au plus, que les Arabes nomment Cassideh ».

\section{8) Sans titre, 2 vers, auteur inconnu}

Le commentaire introductif de Hugo ( «Un autre poëte du divan de Bochteri, recueil de poésies d'hommes inconnus, fleurs du désert dont il ne reste que le parfum, dit:») vient de Fouinet (97r), dont on note au passage la capacité à filer la métaphore. Le « divan de Bochteri» devrait désigner le recueil de poèmes d'al-Buhturī (m. 284/897), mais la présentation qui en est faite comme un «recueil de poésies d'hommes inconnus » montre qu'il s'agit en fait de son anthologie de la poésie archaïque, appelée, comme celle de son mentor Abū Tammām, Hamāsa: c'est la troisième et dernière indication que par « Hamasa », il faut entendre celle d'Abū Tammām. L'anthologie d'al-Buhturī ne comprend pas seulement des extraits anonymes. Mais cette méprise est certainement due au fait que cet extrait provient aussi de Reiske (1742) : il s'agit des deux premiers de sept vers cités p. 82-83 comme venant à la fois de « D. Hud \& Ham. Bocht. » [i.e. dīwān Hudayl et Hamāsa de Buhturī] et dont l'auteur n'est pas cité. La rime en lām m'a permis de les retrouver dans Kosegarten (1854) : ce sont les deux premiers vers de la pièce $n^{\circ} 82$ (p. 165-166), qui compte bien 7 vers, du poète Mālik b. Hālid. En revanche, je ne l'ai pas retrouvée dans la Ḥamāsa d'al-Buhturī, ni par la rime, ni par le nom du poète : il y a plusieurs Mālik dans cet ouvrage, mais aucun n'est le nôtre. Il peut s'agir d'une confusion de Reiske lui-même, dont on notera qu'il ne donne pas ici, contrairement à son habitude, le $n^{\circ}$ du chapitre de la Hamāsa d'alBuhturī (elle est divisée en 174 chapitres).

\section{Quelles sont les sources de Fouinet?}

S'il m'a été possible de déterminer la source des extraits 1, 2, 3, 4 et 18 (l'édition de la Mu'allaqa de Țarafa par Reiske), ainsi que de l'extrait 17 (la première édition de la Chrestomathie de Sacy), il est beaucoup plus difficile de déterminer celle des extraits les plus nombreux, à savoir le dīwān des Hudayl (en dehors de 3, 4 et 18, qui sortent de Reiske, 6 et 7, puis 15 et 16) et la Hamāsa $(8,9,10,11,12,13,14)$ : à l'époque, ni l'un ni l'autre n'étaient encore édités. La conclusion de la note de Hugo met sur une piste : « Nous n'avons point

16 96r-96v-97r-97v constituent bien une seule et même lettre (partiellement lue et transcrite par Martineau), commençant par « Monsieur et ami » et se terminant par « votre ami, Ernest Fouinet ». On ne voit rien sur 97v, sinon 97r en transparence. Cette lettre contient les extraits 17 et 18. 
cherché à mettre d'ordre dans ces citations. C'est une poignée de pierres précieuses que nous prenons au hasard et à la hâte dans la grande mine d'Orient ». Or, Mines de l'Orient, au pluriel, est le titre français d'une revue dont le titre allemand est Fundgruben des Orients et qui a également un titre arabe. Cette revue, publiée à Vienne par Joseph von HammerPurgstall (1774-1856) - la principale source de Goethe - connut six livraisons entre 1809 et 1818. La troisième section y est dans chacune consacrée à la poésie et on y trouve de nombreuses traductions de poèmes persans, mais aussi turcs et arabes. Cette piste est une impasse : aucun ne se retrouve dans la note des Orientales. Il faut donc chercher ailleurs. Fouinet étant Français et ayant étudié à l'Ecole Spéciale des Langues Orientales Vivantes (familièrement : Langues O), on pense d'abord à la Chrestomathie arabe de Silvestre de Sacy, spécialement composée à l'usage des élèves de cette école. Pourtant, en dehors de Šanfarā, aucun des extraits cités par Fouinet ne se trouve ni dans la première édition (1806), ni dans la seconde édition (1826-1827) de cet ouvrage. On pense aussi à l'Anthologie arabe de Jean Humbert (1792-1851), mais, là encore, rien! S'agissant de la Hamāsa en particulier, on pense enfin à une autre chrestomathie : celle de l'orientaliste néerlandais Albert Schultens (1686-1750), qui, rééditant en 1748 la Grammatica arabica de Thomas van Erpe, dit Erpenius (1584-1624), l'augmente de larges extraits de la Hamāsa. Aucun des 31 extraits qu'il cite ne se retrouve cependant chez Fouinet. Le nombre d'ouvrages de ce type étant relativement limité, je ne puis exclure, en l'état actuel de mes recherches, un recours aux manuscrits. Dans ce contexte, on rappellera que les cours d'arabe avaient lieu à la Bibliothèque nationale (royale à l'époque), laquelle abritait (et abrite toujours) une très importante collection de manuscrits orientaux ${ }^{17}$ et que les élèves de l'époque étaient entraînés à lire les écritures manuscrites. Il me semble que les cinq fragments (dont un non repris par Hugo) du Livre I de la Hamāsa, qui se trouvent dans la même zone, et les quatre fragments de l'extrait 16, appartenant à quatre pièces qui se suivent dans le dīwān des Hudayl, appuient cette hypothèse.

\section{Une anthologie de la poésie arabe archaïque}

On a donc un total de 18 extraits (21, si on considère que 16 consiste lui-même en 4 extraits) allant de deux vers à plus d'une dizaine. Ces extraits sont représentatifs de la poésie arabe archaïque, telle que les orientalistes du temps la concevaient et qui démarquait au premier chef la conception que s'en faisaient les érudits arabes médiévaux. Ils juxtaposent en effet un extrait de la Mu'allaqa de Țarafa, les Mu'allaqāt étant la plus célèbre anthologie de la poésie arabe préislamique, et des extraits de la Hamāsa, la plus célèbre anthologie de la poésie arabe archaïque ; un extrait du dīwān d'Imru' al-Qays et des extraits du dīwān des Hudayl, qui sont les plus célèbres recueils respectivement d'un poète individuel et d'un groupe tribal. Il s'y ajoute un extrait de la Lāmiyyat al-'Arab de Šanfarā, le plus célèbre des

17 Il y a bien à la BnF (département des manuscrits) un manuscrit aš ār al-Hudaliyyīn (recension de Sukkārī), Arabe 3286 et cinq manuscrits de la Hiamāsa d'Abū Tammām (Arabe 3281, 3282, 3283, 3284 et 5677), dont deux avec le commentaire de Tibrīzī (3283 et 3284). Un rapporteur anonyme de cet article, qui juge probable l'utilisation de manuscrits, suggère très justement de vérifier leur date d'entrée à la BnF. 
poètes-brigands et, enfin, la Hamāsa de Buhturī est citée, même si l'extrait donné comme en provenant provient en fait du dìwān des Hudayl.

\section{Fouinet, commentateur de la poésie arabe archaïque}

Je donnerai ici seulement deux exemples, l'un concernant le contexte et l'autre le texte lui-même. L'orientalisme du temps en est encore à la collecte des données et c'est seulement plus avant dans le siècle qu'il les soumettra à la méthode historico-critique. Par suite, il répète purement et simplement ces données, qu'à son tour Fouinet reprend, par exemple en ce qui concerne le sens à donner au surnom Múallaqāt (extrait $\mathrm{n}^{\circ} 1$, La Chamelle, Hugo, 1829, p. 401 ; 83r):

« Tous les sept ans, avant l'islamisme, les poëtes de l'Arabie concouraient en poésie, à une foire célèbre, dans un lieu nommé Occadh. La cassideh ${ }^{18}$ (chant) qui avait été jugée la meilleure obtenait l'honneur d'être suspendue aux murailles du temple à la Mecque ; on a conservé sept de ces poëmes ainsi couronnés. Moallakat veut dire suspendue ».

Cette « explication » est, aujourd'hui encore, couramment présentée dans le monde arabo-musulman comme une "vérité historique ». La plupart des arabisants, cependant, s'appuyant d'ailleurs sur certaines sources orientales (notamment Ibn al-Naḥhās), n'y voient qu'une pieuse légende, s'inscrivant dans un débat théologico-idéologique sur l'origine de l'arabe classique.

Ce que le contexte y perd, le texte en revanche y gagne, témoin l'extrait $n^{\circ} 7$. Après le vers 3 («Je veille pour le regarder; il ressemble à la lampe de l'ennemi, brillant dans une citadelle bien fermée, inaccessible »), Fouinet interrompt une première fois sa traduction par le commentaire suivant (90v-91r) :

« Tout à coup le poète frappé de cette idée de citadelle oublie pour la décrire son sujet principal [barré : j'aime cette brusquerie de transitions. C'est sans doute bien l'opposé de l'art, mais n'est-ce pas le caractère de l'inspiration? c'est du moins le caractère de la poésie orientale]. Je reviens à la citadelle $[. .]$.$» ,$

puis, à nouveau après le vers 7 («Les étoiles tendant vers le couchant semblaient ces blanches vaches sauvages ${ }^{19}$ qui s'enfuient du bord de l'étang, où elles s'abreuvaient ») (91r) :

«Quel beau tableau! du haut d'une tour, il voit les étoiles disparaître devant l'aurore, comme les fauves devant le chasseur, les vaches sont blanches comme les astres : le ciel d'où les astres s'effacent semble un étang : c'est bien l'aube ».

Ce sont ces commentaires que résume Hugo (1829, p. 406) d'une formule : « désordre lyrique ». En fait, Fouinet n'a pas parlé de désordre, mais seulement de brusquerie des transitions (qui présupposent ordre) et bien perçu l'emboîtement des comparaisons.

18 Cette ortographe suggère une prononciation orientale de l'arabe qașīda, caractérisée par une imāla $(a>e)$, le $h$ étant destiné à prévenir une réalisation comme un e muet.

19 Il s'agit de baqar al-waȟš (litt. « bœufs sauvages »), c'est-à-dire d'oryx ou d'antilopes à sabre. Les antilopes appartiennent bien à la famille des bovidés. 
D’une manière générale, Fouinet parle de cette poésie avec sympathie, qui est celle d'un poète pour d'autres poètes. Pareillement, Goethe parle de la poésie préislamique avec une extrême chaleur (Goethe 1999, p. 268-269, puis 274-275).

\section{Fouinet, traducteur de la poésie arabe archaïque}

«Extravagantes », les traductions de Fouinet? On sent dans ce qualificatif employé par Louca (cf. supra, introduction) comme une rémanence de «saïdisme »... A la lecture du premier extrait («La chamelle»), l'auteur de ces lignes, qui en a fait lui-même une traduction (le morceau passe pour « impossible»!), a été au contraire frappé, à la fois par la concision de la traduction de Fouinet (à chaque vers, correspond un verset) et sa relative précision. Afin d'en donner une idée au lecteur non arabisant, je me permets de mettre en regard la traduction de Fouinet et la mienne (LARCHER 2000, p. 62-64) :

\begin{tabular}{|c|c|}
\hline Fouinet & Larcher \\
\hline $\begin{array}{l}\text { Elle est solide comme les planches d'un cercueil, } \\
\text { quand je la pousse sur un sentier frayé, comme un } \\
\text { manteau couvert de raies. }\end{array}$ & $\begin{array}{l}12 \text { Sûre, comme les ais d'un grand cercueil, je l'active } \\
\text { Dessus le vêtement rayé du grand chemin. }\end{array}$ \\
\hline $\begin{array}{l}\text { Elle dépasse les plus rapides, et rapidement son pied } \\
\text { de derrière chasse son pied de devant. }\end{array}$ & $\begin{array}{l}\text { 14 Elle défie les meilleures, à la course, faisant suivre } \\
\text { A sa jambe sa jambe sur la piste damée. }\end{array}$ \\
\hline $\begin{array}{l}\text { Elle obéit à la voix de son conducteur, et, de sa queue } \\
\text { épaisse, elle repousse les caresses violentes du } \\
\text { chameau au poil roux; }\end{array}$ & $\begin{array}{l}16 \text { Elle revient à l'appel et se garde, d'une queue } \\
\text { Touffue, des terreurs d'un mâle, au poil brun feutré : }\end{array}$ \\
\hline $\begin{array}{l}\text { D'une queue qui semble une paire d'ailes d'aigle que } \\
\text { l'on aurait attachées à l'os avec une alêne; }\end{array}$ & $\begin{array}{l}17 \text { On dirait les deux ailes d'un aigle blanc, couvrant } \\
\text { Ses flancs, fichés dans l'os caudal par une alêne! }\end{array}$ \\
\hline $\begin{array}{l}\text { D'une queue qui frappe tantôt le voyageur, tantôt } \\
\text { une mamelle aride, tombante, ridée comme une } \\
\text { outre. }\end{array}$ & $\begin{array}{l}18 \text { Tantôt, elle en bat, derrière, l'ami, et tantôt } \\
\text { L'outre desséchée de son pis, flétri, tari. }\end{array}$ \\
\hline $\begin{array}{l}\text { Ses cuisses sont d'une chair compacte, pleine et } \\
\text { ressemblent aux portes élevées d'un château fort. }\end{array}$ & $\begin{array}{l}19 \text { Deux cuisses à la chair si parfaite, qu'on dirait } \\
\text { Les vanteaux du portail d'un palais haut et lisse, }\end{array}$ \\
\hline $\begin{array}{l}\text { Les vertèbres de son dos sont souples; ses côtes } \\
\text { ressemblent à des arcs solides. }\end{array}$ & $\begin{array}{l}20 \text { Le rouleau des vertèbres, l'arceau des apophyses, } \\
\text { L'encolure au garot, qui colle et s'articule! }\end{array}$ \\
\hline $\begin{array}{l}\text { Ses jambes se séparent quand elle court, comme les } \\
\text { deux seaux que porte un homme du puits à sa tente. }\end{array}$ & $\begin{array}{l}22 \text { Deux coudes bien arqués, comme elle transportant } \\
\text { Les deux seaux, pris au puits, d'un solide abreuveur ; }\end{array}$ \\
\hline $\begin{array}{l}\text { Les traces des cordes sur ses flancs semblent des } \\
\text { étangs desséchés et remplis de cailloux épars sur une } \\
\text { terre aride. }\end{array}$ & $\begin{array}{l}27 \text { Les traces, sur son corps, des sangles sont ruisseaux } \\
\text { Surgis d'un rocher lisse, dessus un rocher dur, }\end{array}$ \\
\hline $\begin{array}{l}\text { Son crâne est dur comme l'enclume; celui qui le } \\
\text { touche croit toucher une lime. }\end{array}$ & $\begin{array}{l}30 \text { Son crâne est une enclume, dont les bords, dirait-on, } \\
\text { Là où ils jointent, sont joints au rebord d'une lime ; }\end{array}$ \\
\hline $\begin{array}{l}\text { Sa joue est blanche comme du papier de Damas, ses } \\
\text { lèvres noirâtres comme du cuir du Yémen, dont les } \\
\text { courroies ne se rident point. }\end{array}$ & $\begin{array}{l}31 \text { Sa joue, un parchemin de Syrie ; sa babine, } \\
\text { Une peau du Yémen, proprement découpée ; }\end{array}$ \\
\hline $\begin{array}{l}\text { Enfin elle ressemble à un acqueduc, dont le } \\
\text { constructeur grec a couvert de tuiles le sommet. }\end{array}$ & $\begin{array}{l}23 \text { Comme arche romaine, que son maître d'oeuvre adjure } \\
\text { D'échafauder bien, pour la rehausser de briques ; }\end{array}$ \\
\hline
\end{tabular}


On se souvient que Hugo, à la fin de son introduction des fragments, écrit, après avoir mentionné Fouinet: "Nous conservons scrupuleusement sa traduction; elle est littérale et par conséquent, selon nous, excellente ». Hugo n'a évidemment pas les moyens de juger. Il a en fait résumé d'une phrase ce que Fouinet dit de sa propre traduction (à la suite de l'extrait $\left.n^{\circ} 7,91 v\right)$ :

«j'ai traduit tout ceci fort négligemment quant au style qui est plus arabe que français, mais c'est ce qu'il faut; l'exactitude y est. Comptez-y et regardez ce que je vous fais connaitre comme votre propriété exclusive : il serait beau pour moi de pouvoir vous enrichir. Tous les renseignements qui vous seraient utiles, demandez-les moi et je vous les donnerai si j'en suis capable. C'est une faveur que vous me rendrez en me forçant à ne pas oublier la clef de mes trésors de poésie ».

Albouy, dans son édition des œuvres poétiques de Hugo (1964, p.1327), sans malheureusement citer de source, écrit :

«En fait, Fouinet a traduit cette pièce de la Chamelle, celles de la Cavale, de la Traversée du désert pendant la nuit, de Pendant le jour, non pas de l'original, mais de la traduction latine de la Moallakah de Tarapha, publiée par Jean-Jacques Reiske, à Leyde, en $1742{ }^{20}$.

L'affirmation est un peu rapide, car même si Fouinet a un œil sur le latin, il en a bien un autre sur l'arabe, comme le montre indubitablement le premier vers de la troisième de ces pièces (d'Umayya b. Abī al-'Á'id, p. 87) :

Umayya : wa-ğawwābu ğawwāti l-fiğāği llatī bihā/n-na'āmu wa-'azfu l-ğinni wa-l-mutaġawwilū (" Grand traverseur de profonds défilés, où sont / Autruche, sifflement des djinns, avatar de goule...»)

Reiske: Ego sum pervasor per curva amfractuum praecipitia, in quibus struthio-cameli et sibilus Satyrorum et Empusa

Fouinet: Je me plonge dans les anfractuosités des précipices, dans des solitudes où sifflent les djinns et les gouls

Fouinet rétablit djinns et goule que Reiske a transculturés en satyres et empuse ! J'avais d'abord supposé, en lisant cette note d'Albouy, qu'un arabisant était intervenu. Il me semble maintenant que soit Albouy, soit sa source ont consulté Reiske, découvert qu'il était non seulement la source du premier extrait, mais encore des trois suivants, en collationnant la traduction de Fouinet à celle en latin de Reiske, non à l'original arabe.

En un endroit, enfin, Hugo censure Fouinet. Le vers 5 de « La cavale » se termine par des points de suspension, assortis d'une note $\left(n^{\circ} 2\right):$ : il y a ici quelque chose de primitif et qui pourrait tout au plus se traduire en latin ». Ce quelque chose est en fait le second hémistiche (tasuddu bihi farğahā min duburin, litt. " par laquelle elle barre sa fente par derrière ») parfaitement traduit en latin par Reiske (qui obstruit vulvam a postica) : la poésie française de l'époque avait des pudeurs que n'avaient ni l'antique poésie arabe ni ses interprètes européens en latin du XVIII ${ }^{e}$ siècle, ni même Ernest Fouinet qui dans sa lettre

20 Repris par Albouy dans son édition des Orientales (Hugo, 1966, p. 368) sous la forme suivante : « Les quatre premières pièces de cette note ont été traduites, non pas de l'original, comme l'écrit Hugo, mais de la traduction latine de la Moallakah de Tarapha (Leyde, 1742)». 
traduit $(84 \mathrm{v})$ « elle sépare sa vulve de son fondement », ajoutant, entre parenthèses, « ceci est tout à fait primitif ».

\section{Arabes vs Persans}

«Des Arabes aux Persans la transition est brusque ; c'est comme une nation de femmes après un peuple d'hommes. Il est curieux de trouver, à côté de ce que le génie a de plus simple, de plus mâle, de plus rude, l'esprit, rien que l'esprit, avec tous ses raffinements, toutes ses manières efféminées. La barbarie primitive, la dernière corruption; l'enfance de l'art et sa décrépitude. C'est le commencement et la fin de la poésie qui se touchent. Au reste, il y a beaucoup d'analogie entre la poésie persane et la poésie italienne. Des deux parts, madrigaux, concettis, fleurs et parfums. Les Persans sont les Italiens de l'Asie.»

Ainsi Hugo (1829, p. 414) introduit-il les extraits de poèmes persans, qui suivent les poèmes arabes. La collation de la version imprimée des Orientales avec le manuscrit $(99 \mathrm{r}-101 \mathrm{v})$ montre que Fouinet donnait en fait neuf extraits et dans un ordre différent: $1=4$ de Hugo ; 2 = 1 de Hugo ; $3=6$ de Hugo ; 4, non repris, est un morceau d'Asadi ainsi présenté : « Dans une dispute de préséance, le jour et la nuit disent plusieurs raisons dont quelques-unes méritent d'être rapportées »; $5=2$ de Hugo; $6=3$ de Hugo ; 7 et 8, non repris, sont deux morceaux de Saadi, respectivement un quatrain et un récit ; $9=5$ de Hugo.

Les deux déplacements s'expliquent aisément: ont été réunis les deux morceaux de Firdoussi ( 5 et 6 de Hugo) et les deux morceaux de poètes mystiques, Djelal Eddin Roumi et Ferideddin Attar (3 et 4 de Hugo).

La même collation montre que si les commentaires factuels viennent directement de Fouinet, le chapeau introductif reprend, sinon la lettre, du moins l'esprit d'un commentaire de Fouinet. Hugo y oppose la poésie persane à la poésie arabe comme une poésie féminine à une poésie virile, une poésie de peuple dominé, osant une comparaison avec l'Italie. Or, Fouinet, après le fragment $n^{\circ} 5$ (de Chahpour Abhari) commente (100r-100v) :

«La pièce précédente [i.e. celle d'Asadi, non reprise], celle-ci surtout [i.e. celle de Chahpour Abhari], n'ont-elles pas le caractère de la poésie italienne, comme le persan avec le caractère italien? Ce qu'il y a de plus remarquable, c'est que les deux peuples ont toujours été dans une position de dépendance qui paraît avoir également influé sur eux. »

C'est donc Fouinet qui esquisse un trait que Hugo grossit jusqu'à la caricature.

Fouinet ajoute (100v) : " Mais assez d'antithèses ; voici un Ghazel d'une vraie beauté », phrase qui introduit l'extrait de Djelal Eddin Roumi. Hugo la reprend, mais en ajoutant "d'une beauté arabe... », qui résume sa conception de l'opposition des deux poésies. Estce un hasard ? Ces cinq vers de Roumi sont cités par Jules Michelet (1798-1874) dans son Histoire de France (t. II, p. 314, Paris, Hachette, 1833), comme ceux d'un auteur... arabe (!). Michelet introduit et conclut sa citation par ces mots :

«Voici un passage d'un auteur arabe, qui offre, avec les idées exprimées par Saint Bernard, une remarquable analogie : (...). (Ce beau fragment, dû à un jeune orientaliste, M. Ernest Fouinet, a été inséré par M. Victor Hugo dans les notes de ses Orientales, p. 416 de la première édition). » 
Le contexte de la citation est intéressant. Évoquant le roi Louis VII et la seconde croisade, prêchée par Saint Bernard de Clairvaux (1090 ou 1091-1153), mais que ce dernier refusa d'accompagner, Michelet renvoie à une lettre, très antérieure, de Bernard à l'évêque de Lincoln qui s'enquérait des nouvelles d'un chevalier anglais parti pour la Terre Sainte et qui, faisant halte à Claivaux, y était resté. Bernard justifie l'interruption du voyage par le fait que la Jérusalem à conquérir n'est pas la Jérusalem terreste, mais céleste ! Pareillement, Djelal Eddin Roumi conclut son poème sur la Caaba ainsi : « Ô adorateurs d'une maison! pourquoi adorer de la pierre et de la boue! Adorez l'autre maison, celle que cherchent les élus ».

On doit admirer Michelet, non seulement pour ce rapprochement inattendu, mais encore pour l'histoire singulièrement moins rustique des Croisades (Saint Bernard, prêchant une croisade à laquelle il ne croit pas...) que celle qu'on nous sert aujourd'hui.

\section{Le paradoxe}

Le paradoxe est que les extraits de poèmes orientaux fournis par Fouinet à Hugo n'ont eu sur Hugo lui-même que très peu d'influence ${ }^{21}$ et que celle qu'ils ont eue sur la poésie française ultérieure semble en raison inverse de leur importance numérique: les plus nombreux, les poèmes arabes, paraissent en effet, sous bénéfice d'inventaire, en avoir eu moins que les poèmes persans, pourtant moins nombreux, et ceux-ci encore moins que l'unique « pantoum malais ${ }^{22}$.

Hugo lui-même ne revendique une influence directe que dans deux cas: le poème «Adieux de l'hôtesse arabe » (XXIV) et le poème « Mazeppa» (XXXIV). Après les quatre premiers vers de la Cavale (extrait $n^{\circ} 2$ ) qui sont :

«La cavale qui m'emporte dans le tumulte a les pieds longs, les crins épars, blanchâtres, se déployant sur son front.

Son ongle est comme l'écuelle dans laquelle on donne à manger à un enfant. Il contient une chair compacte et ferme.

Ses talons sont parfaits, tant les tendons sont délicats.

Sa croupe est comme la pierre du torrent qu'a polie le cours d'une eau rapide. »

Hugo ajoute une note indiquant :

(1) L'auteur a traduit ce passage dans les Adieux de l'hôtesse arabe: Ses pieds fouillent le sol, sa croupe est belle à voir, Ferme, ronde et luisante, ainsi qu'un rocher noir, Que polit une onde rapide.

21 Cela s'explique facilement par des raisons de chronologie. Hugo a commencé la composition des Orientales dès 1825, alors que Fouinet n'intervient qu'en 1828 (Stéphane Baquey, communication personnelle).

22 En fait pantoun : l'examen du manuscrit (101v) suggère que c'est l'imprimeur qui a pris pour un $m$ ce qui est en fait un $n$, cf. conclusion. 
De même, après le cinquième vers de «Pendant le jour » (extrait $n^{\circ} 4$ ), qui est: "De même je m'enfonce dans une plaine poussiéreuse, dont le sable agité ressemble à un manteau rayé », Hugo ajoute une note indiquant :

(1) Cette belle et pittoresque expression a été traduite par l'auteur dans cette strophe de Mazeppa:

\author{
Et si l'infortuné, dont la tête se brise, \\ Se débat, le cheval qui devance la brise, \\ D'un bond plus effrayé \\ S'enfonce au désert, vaste, aride, infranchissable, \\ Qui devant eux s'étend avec ses plis de sable, \\ Comme un manteau rayé.
}

Le terme « traduit », qu'emploie par deux fois Hugo, mériterait tout un commentaire... Un Français scolarisé de ma génération qui a appris la littérature française, classe après classe et siècle après siècle, dans le bon vieux Lagarde et Michard, ne se souvient pas d'y avoir rencontré, pour le XIX siècle, un poème à la manière arabe ou persane. S'il en existe, aucun n'est devenu « classique ». En revanche, il se souvient au moins d'un pantoum : Harmonie du soir de Charles Baudelaire (1821-1867), dont la célébrité, certainement due à l'école, est confirmée par le fait qu'il est la première des douze Fleurs du mal mises en musique (1957) par Léo Ferré (1916-1993), ce qui a contribué à le rendre plus célèbre encore ${ }^{23}$. Bien sûr, du pantoum ou plutôt d'une variété de celui-ci, le poète français n'a retenu que la forme, c'est-à-dire la reprise des premier et troisième vers du premier quatrain comme deuxième et quatrième du second quatrain (et ainsi de suite), sans en retenir la structure (deux thèmes). Il n'empêche : c'est bien là une influence directe et manifeste des Notes des Orientales sur la poésie française ultérieure. Aujourd'hui, seuls les spécialistes savent que Leconte de l'Isle (1818-1894) a inclus cinq pantoums dans ses Poèmes Tragiques (1884) et que Théodore de Banville (1823-1891) en traite dans son Petit traité de poésie française (1871). Dans sa présentation des Orientales, Meschonnic rappelle que dans le poème Erythrea des Chimères (1854) de Gérard de Nerval (1808-1855), les vers : «Car voici le Vautour volant sur Patani, / Et de papillons blancs la mer est inondée », sont une réminiscence du pantoum des Orientales fourni par Fouinet : «Les papillons jouent à l'entour sur leurs ailes... Le vautour dirige son essor vers Bandam... Et laisse tomber de ses plumes à Patani »...

\title{
Conclusion
}

L'assertion de Schwab, citée en introduction, ne nous paraît donc pas très adéquate, et pas seulement sur le plan géographique et chronologique, Fouinet fournissant en effet des extraits de la poésie arabe archaïque, tant préislamique que des premiers temps de l'islam, de la poésie persane et de la poésie malaise. En fait, malgré Hugo relayant Fouinet, la qașìda arabe (la « cassideh » sous leur plume) semble bien rater son entrée sur la scène

23 C'est également un des Cinq poèmes de Baudelaire (1887-1889), mis en musique par Claude Debussy (1862-1918). 
littéraire. Pourquoi ? Peut-être parce que Hugo n'a pas fait ce que Goethe a fait : adapter à la poésie nationale un poème arabe. Pour faire passer en allemand le chant de vengeance de Ta'abbața Šarran, Goethe a choisi une des grandes formes de la poésie allemande (et, notamment, du Volkslied), le quatrain à rythmes libres. Quelle forme aurait pu choisir Hugo, sinon celle où il excella plus tard, la grande ode en alexandrins et à rimes plates, profondément accordée au ton généralement épique de la qașīda et que laissent entrevoir les traductions de Fouinet ${ }^{24}$ ? À la décharge de Hugo, on peut dire qu'il était alors un jeune poète, presque encore à ses débuts (Les Orientales sont, après Odes et Ballades (1826), son deuxième recueil), tandis que Goethe était un vieux poète, dont le Divan est en fait le dernier recueil ${ }^{25}$... Peut-être aussi parce que Fouinet n'a pas fait ce qu'un autre « écrivain de savoir et d'imagination », Friedrich Rückert (1788-1866), a fait pour la poésie orientale en général et la poésie arabe archaïque en particulier, à travers ses traductions du dīwān d'Imru' al-Qays (Amrilkais, 1843) et de la Hamāsa (1846), imposant le distique rimé. À la décharge de Fouinet, on peut dire qu'il a essayé. Selon Schwab (1950, p. 352), Fouinet reprit l'année suivante quelques-unes des pièces citées par Hugo dans un ouvrage collectif, dirigé par Francisque Michel (1809-1887), le futur grand éditeur de textes médiévaux, et où l'on trouve aussi des traductions d'autres orientalistes. Schwab n'a pas consulté l'ouvrage. S'il l'avait fait, il aurait vu d'abord que Michel (Liminaire, p. VIII-IX) remercie Fouinet, comme étant avec l'orientaliste Joseph Toussaint Reinaud (1795-1867), une des deux chevilles ouvrières de l'ouvrage. Il le fait en ces termes :

«M. Ernest Fouinet, jeune poète déjà honorablement connu par une foule d'ouvrages ${ }^{26}$ où éclate un talent haut, varié et prometteur de grandes choses, et par la traduction des poésies arabes dont il a enrichi les Orientales de Victor Hugo, et, de plus, possesseur d'une profonde érudition, M. Fouinet a bien voulu, avec un rare désintéressement, consacrer de longues et pénibles veilles à la traduction en vers des morceaux malais et persans qui portent son nom, et qui sont un des plus beaux ornements de notre recueil. »

Il aurait vu aussi que, parmi les pièces malaises (p.37-64), Fouinet donne en décasyllabes (quatre strophes de quatre vers) le pantoun (ainsi orthographié) des Orientales, en indiquant au passage la source, la "Grammaire de la langue malaise de M. Marsden » [i.e. Grammar and Dictionary of the Malay Language (1812) de William Marsden (1754-1836)], ainsi qu'un autre poème malais, en alexandrins (« Mort de Keni Tambouhan », p. 47-57)

24 C'est par «ode » que l'on «traduit » généralement qașīda. Les mètres les plus usuels de la poésie archaïque font, en décompte syllabique, deux syllabes de plus que notre alexandrin.

25 La jeunesse de Hugo doit bien être incriminée. Stéphane Baquey (communication personnelle) me confirme que les Orientales sont sa période «strophique », et, plus largement, de recherches formelles, dont témoigne l'époustouflant Les Djinns.

26 Si l'on en croit le catalogue de la BnF, Fouinet n'avait alors publié que Psara, élégie épique (Paris, Delaunay, 1824, in- $\left.8^{\circ}, 12 p\right)$. C'est très certainement un poème sur le massacre perpétré par les Ottomans en Juillet 1824 sur l'île grecque de Psara (17000 victimes, morts et captifs réduits en esclavage, dit-on), mais moins connu que celui de Chio, deux ans auparavant. Cela vient rappeler que le contexte premier des Orientales est celui dit du « philhellénisme », c'est-à-dire du soutien de l'Europe (en fait de ses élites intellectuelles) à la guerre d'indépendance de la Grèce (au siècle suivant on dira : « de libération nationale »). La «foule d'ouvrages » sont certainement un certain nombre de poèmes parus dans la presse. 
et un autre pantoun (cinq strophes de quatre vers, trois décasyllabes et un tétrasyllabe, p. 61-62). Il aurait vu enfin et surtout que Fouinet ne reprend aucune des pièces arabes citées par Hugo. Il fait bien mieux. Il traduit en vers plusieurs pièces célèbres de la poésie arabe : tout d'abord, sous l'appellation « Le Chant de mort » (p. 127-130), le poème même de Ta'abbața Šarran adapté par Goethe dans son Divan ${ }^{27}$; puis « La moallakat de Libid [sic] » (p. 227-236), ce qui le révèle moins révérencieux à l'égard du maître, Silvestre de Sacy, qui l'avait traduite en prose (SILVESTRE DE SACY 1816), que son élève Armand-Pierre Caussin de Perceval (1795-1871), qui ne traduira que six des sept Mu'allaqāt, se contentant de donner celle de Labīd dans la version de Silvestre de Sacy (CAUSSIN DE PERCEVAL 1847-1848) ${ }^{28}$; et, enfin, sous l'appellation de «Tableau d'une station abandonnée » (p. 247-249), le début de la Moallakat de Boheïr [sic], i.e. Zuhayr. Et la forme poétique adoptée par Fouinet est bien celle attendue : celle de l'ode continue en alexandrins et à rimes plates, seulement discontinuée, ici ou là, par un espacement, pour signaler les grandes « parties » du poème arabe... Tout cela donne à penser que Fouinet ambitionnait de «naturaliser » la poésie orientale, non seulement arabe, mais encore persane et malaise. Cet ouvrage, toutefois, ne semble pas avoir eu de succès, comme le suggère le fait que seul le tome I est paru et qu'on le trouve dans très peu de bibliothèques ${ }^{29}$. Ceci explique (peut-être) ${ }^{30}$ cela : que Fouinet ait délaissé l'Orient, poursuivant jusqu'à sa mort une double carrière de fonctionnaire des finances et d'homme de lettres, poète et romancier, notamment pour la jeunesse, mais où on ne trouve que deux productions orientalisantes : La Caravane des morts (1836) et les Anémones du Roi Noman (1855) ${ }^{31}$. Pertuis (1924) a publié plusieurs lettres de Fouinet à Hugo,

27 C'est d'autant moins un hasard que dans la « notice sur la pièce suivante » qui précède la pièce, Fouinet (p. 124) indique qu' «il est d'une poésie sombre, sinistre, nocturne, comme l'a dit Goethe de ce même morceau ». Pour Fouinet, Ta’abbața Šarran n'est pas l'auteur du poème, mais la victime vengée dans ce poème, le vengeur étant un de ses neveux. La mention de l'édition en cours de la Hamāsa (cf. supra 1) montre que Fouinet est informé de ce qui se fait, en matière d'orientalisme, outre-Rhin.

28 Dans la notice qui précède (p. 222), Fouinet écrit cependant: « Ces courts renseignements sont extraits de la Notice sur Libid, que l'illustre professeur Silvestre de Sacy a donné en tête de sa Moallakat, dont il a publié le texte et le commentaire, en y joignant une traduction littérale en prose. Quoique travaillant sur le texte arabe, j'ai tiré tout le fruit possible des savantes interprétations de M. de Sacy qu'une étude longue, intelligente, approfondie de la langue pouvait seule produire ». Ce qui ne l'empêche pas d'ajouter (p. 224): «Voici les conditions de la traduction d'une langue à l'autre ; elles sont sévères : puissé-je les avoir observées et avoir réussi dans mes vers : on sentira d'après les explications qui précèdent, combien ma tâche était difficile :j'ai cru devoir l'aborder sans crainte, persuadé qu'un poète ne peut être rendu que par la poésie, dans quelque langage que ce soit », concluant: «L'art n'est rendu que par l'art ».

29 En dehors de la BnF, il ne se trouve, selon SUDOC, qu'à Paris-BULAC, où je l'ai consulté, les 29 mai et 11 juin 2012. Les deux tomes sont ainsi présentés dans le Liminaire, p. XI : « Le premier volume de ces poésies contient des pièces traduites de sanskrit, javanais, malais, chinois, thibétain et arabe. Le second, qui suivra de près celuici, renfermera des traductions de poésies persanes, georgiennes [sic], arméniennes, turques et indoustannes, qui donneront lieu à un nouvel et court avant-propos ».

30 Plus vraisemblablement, Fouinet, orientaliste amateur et non professionnel, sans poste à l'Université, s'est tourné vers une littérature plus alimentaire...

31 Le plus connu est Gerson ou le manuscrit aux enluminures (1842), qui connut de nombreuses rééditions jusqu'à la fin du XIX siècle. Le catalogue général de la BnF donne bien d'autres titres, dont un certain nombre disponibles sur Gallica. Un collègue de l'Université d'Artois, Francis Marcoin, s'intéresse à cette partie de l'œuvre de Fouinet (Stéphane Baquey, communication personnelle). 
datant des années 1840, où Fouinet sollicite Hugo d'intervenir en sa faveur pour l'obtention d'un prix littéraire, le prix Montyon. Il obtiendra le $3^{\mathrm{e}}$ prix (le premier allant à une amie de Hugo !), avant de disparaitre, jeune encore, en 1845.

Il connaissait Sainte-Beuve (1804-1869) et Sainte-Beuve le connaissait, qui lui dédie un des poèmes des Consolations (1830), le n VIII, daté d'Août 1829 (Sainte-Beuve, 1840, p. 194196). Pourquoi Sainte-Beuve, alors jeune homme (il a vingt-cinq ans) dédie-t-il un poème aussi désabusé à un homme jeune (Fouinet en a trente) ? Je l'ignore. Espérons seulement qu'il n'était pas prémonitoire : «Homme, vivre ignoré, modeste... ».

Même s'il n'a pas persévéré, même s'il n'a pas fait école, Ernest Fouinet n'en apparaît pas moins comme un authentique pionnier en matière de traduction de la poésie arabe archaïque. Il est juste de s'en souvenir.

\section{Bibliographie}

\section{Euvres de Victor Hugo}

Hugo Victor, Les Orientales, manuscrit, Paris, Bibliothèque nationale de France, Département des manuscrits, NAF 13359. [En ligne] http://gallica.bnf.fr/ark:/12148/btv1b60008362. $\mathrm{r}=$ victor+hugo\% $2 \mathrm{C}+$ orientales.langFR

Hugo Victor, 1829, Les Orientales, cinquième édition, dans CEuvres de Victor Hugo, tome III, Paris, Charles Josselin et Hector Bossange. [En ligne] http://gallica.bnf.fr/ark:/12148/btv1b8626456q. $\mathrm{r}=$ victor+hugo\% $2 \mathrm{C}+$ orientales.langFR

Hugo Victor, 1952-1954, Les Orientales, édition critique avec une introduction, des notices, des variantes et des notes par Elisabeth Barineau (Société des textes français modernes), Paris, Didier, 2 tomes.

Hugo Victor, 1964, CEuvres Poétiques I Avant l'exil 1802-1851, préface par Gaëtan Picon, édition établie et annotée par Pierre Albouy, Paris, Gallimard (Bibliothèque de la Pléiade).

Hugo Victor, 1966, Les Orientales. Les Feuilles d'automne, édition présentée, établie et annotée par Pierre Albouy, Paris, Gallimard (collection Poésie).

Hugo Victor, 1970, Les Orientales, présentation par Henri Meschonnic, dans Victor Hugo, CEuvres Complètes, édition chronologique publiée sous la direction de Jean Massin, tome troisième, p. 431-604, Paris, Le Club Français du Livre.

Hugo Victor, 2000, Les Orientales. Les Feuilles d'automne, présentation, notes et dossier par Franck Laurent, Paris, Librairie Générale Française (Le Livre de Poche classique).

\section{Sources arabes}

Abū Tammām, Hamāsa, voir Tibrī̄ī.

Bag̉dādī, 'Abd al-Qādir b. 'Umar al-, Hizānat al-adab wa-lubb lubāb lisān al-'Arab, éd. 'Abd al-Salām Muḥammad Hārūn, Le Caire, al-Hay'a al-mișriyya al-'āmma li-l-kitāb, 1399/1979, 13 vol.

Buhturī, Abū 'Ubāda al-, al-Hạā̄sa, éd. Kamāl Muștafā. Le Caire, al-Maktaba al-tiğāriyya al-kubrā, 1929. Ibn Hišām, Abū Muhammad 'Abd Allāh, Sīrat al-nabī, éd. Muḥammad Muḥȳ̄ al-Dīn 'Abd al-Ḥamīd, 4 vol. [réimpression Dār al-Fikr, 1401/1981]. 


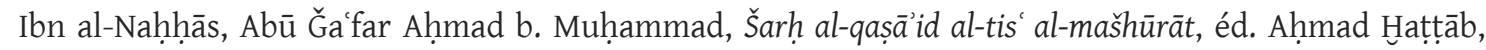
Bagdad, Dār al-ḥurriyya et Maṭba at al-ḥukūma, 1393/1973, 2 vol.

Tibrīzī, Abū Zakariyyā' Yahyā b. 'Alī al-, Šarh Dīwān al-Hamāsa li-Abī Tammām, Būlāq, 1296H, 4 vol.

Zawzan̄i, Abū 'Abd Allāh al-Ḥusayn b. Ahmmad b. al-Ḥusayn al-, Šarh al-Mu'allaqāt al-sab', éd. Muhammad 'Alī Ḥamd Allāh, Damas, al-Maktaba al-umawiyya, 1383/1963.

\section{Autres références}

AHLWARDT Wilhelm, 1870 [1913], The divans of the six ancient Arabic poets. Ennâbigha, 'Antara, Tharafa, Zuhair 'Alqama and Imruulqais; chiefly according to the MSS of Paris, Gotha and Leyden and the Collection of their Fragments, with a list of the various Readings of the Text, edited by W. Ahlwardt, London, Trübner \& Co. [réimpression, Paris, Geuthner].

BLACHÈRE Régis, 1952-1964-1966, Histoire de la littérature arabe des origines à la fin du XVe siècle, Paris, Librairie d'Amérique et d'Orient Adrien-Maisonneuve, 3 vol.

CAUSSIN DE PERCEVAL Armand-Pierre, 1847-1848, Essai sur l'histoire des Arabes avant l'islamisme, pendant la période de Mahomet et jusqu'à la réduction de toutes les tribus sous la loi musulmane, Paris, Firmin Didot frères, 3 vol.

FREYTAG Georg Wilhelm, 1828, Hamasae Carmina cum Tebrisii scholiis integris primum editit, indicibus instruxit, versione latina et commentario illustravit Georg. Guil. Freytag (...). Pars prior, continens textum arabicum et quatuor indices, Bonnae Typis Regiis Arabicis in officina Baadeni.

FREYTAG Georg Wilhelm, 1847-1851, Hamasae Carmina cum Tebrisii scholiis integris edita, versione latina commentarioque illustravit et indicibus instruxit Georg. Guil. Freytag (...). Pars posterior et Pars posterior II, continens versionem latinam, commentarium et indices, Bonnae in officina Lechneri, venditur apud marcum.

Fundgruben des Orients, 1809-1818, bearbeitet durch eine Gesellschaft von Liebhabern / Mines de l'Orient, exploitées par une société d'amateurs / Mahzzan al-kunūz al-mašriqiyya wa-ma'din al-rumūz alağnabiyya, ta'līf ğamā'a min 'udabā', Wien, Anton Schmid, tomes I-VI.

GOETHE Johann Wolfgang, 1999, West-östlicher Divan, Studienausgabe, herausgeben von Michael Knaupp, Stuttgart, Reclam.

HUMBERT Jean, 1819, Anthologie arabe, ou Choix de poésies arabes inédites, traduites en français, avec le texte en regard, et accompagnées d'une version latine littérale, par Jean Humbert, Paris, Treuttel et Würtz.

KOSEGARTEN Johann Gottfried Ludwig, 1854, Carmina Hudsailitarum, quotquot in codice Lugdunensi insunt, arabice edita adiectaque translatione adnotanionibusque illustrata ab Joanne Godofredo Ludovico Kosegarten (...).Volumen primum. Sumtu Societatis Anglicae quae Oriental Fund nuncupatur. Prostat Gryphisvaldiae in libraria C. A. Kochiana, Th. Kunice, Londonii apud W.H. Allen, Lutetiae apud B. Duprat/The Hudsailian Poems, contained in the manuscript of Leyden, edited in Arabic and translated with annotations by John Godfrey Lewis Kosegarten (...). Vol. I, containing the first part of the Arabic text. London, printed under the patronage of the Oriental Translation Fund of Great Britain and Ireland. Sold by Wm. H. Allen et C ${ }^{\circ}$, Leadenhall Street. B. Duprat, Paris, 1854.

LARCHER Pierre, 2000, Les Mu'allaqât. Les sept poèmes préislamiques, préfacés par André Miquel, traduits et commentés par Pierre Larcher, Saint-Clément de Rivière, Fata Morgana (collection Les immémoriaux).

LARCHER Pierre, 2012, «Goethe et Ta’abbața Šarran, une fois encore », Arabica 59/1-2, p. 157-163.

LAROUSSE Pierre, 1866-1877, Grand dictionnaire universel du XIXe siècle, français, historique, géographique, mythologique, bibliographique, littéraire, artistique, scientifique, etc., Paris, Administration du grand dictionnaire universel, 17 vol. 
LOUCA Anouar, 2001, « Les sources marseillaises de l'Orient romantique », Paris, Maisonneuve et Larose (collection Victor Hugo et l'Orient).

LOUCA Anouar, 2006, L'autre Égypte, de Bonaparte à Taha Hussein, Le Caire, Institut Français d'Archéologie Orientale.

MAC GUCKIN DE SLANE William, 1837, Le Diwan d'Amro'lkaïs, précédéde la vie de ce poëte par l'auteur du Kitab elAghani, accompagné d'une traduction et de notes par le $B^{\text {on }}$ Mac Guckin de Slane, Paris, Imprimerie royale.

MARTINEAU René, 1916, «Ernest Fouinet et les “Orientales” », Mercure de France 115, p. 648-659.

MICHEL Francisque, éd., 1830, Choix de poésies orientales; traduites en vers et en prose par MM. Ernest Fouinet, Garcin de Tassy, Grangeret de la Grange, Humbert, Klaproth, Langlès, G. Pauthier, Reinaud et Silvestre de Sacy; recueillies par Francisque Michel, Paris, de l'imprimerie de Béthune, rue Palatine $\mathrm{n}^{\circ} 5$, tome premier.

MiCHELET Jules, 1833-1841, Histoire de France, Paris, Hachette, tomes I-V.

PERTUIS Gervais, 1924, "Autour d'un prix littéraire. Victor Hugo et Ernest Fouinet, avec une correspondance inédite », Mercure de France 159, p. 410-423.

REISKE Johann Jakob, 1742, Tharaphae Moallakah cum Scholiis Nahas. e MSS Leidensibus Arabice edidit, vertit, illustravit Johann. Jacob. Reiske, Lugduni Batavorum, apud Joannem Luzac, MDCCXLII.

RÜCKERT Friedrich, 1843, Amrilkais, der Dichter und König. Sein Leben dargestellt in seinen Liedern. Aus dem Arabischen übertragen von Friedrich Rückert, Stuttgart und Tübingen, Gotta'scher Verlag.

RÜCKERT Friedrich, 1846, Hamâsa oder die ältesten arabischen Volkslieder, gesammelt von Abu Temmâm, übersetzt und erläutert von Friedrich Rückert, Stuttgart, Samuel Gottlieb Liesching, 2 parties.

SCHWAB Raymond, 1950 [1948], La Renaissance orientale, Paris, Payot [texte remanié de La Renaissance orientale (1771-1870), thèse de doctorat, Paris, Université de la Sorbonne].

SAINTE-BEUVE Charles-Augustin, 1840, Poésies complètes de Sainte-Beuve. Joseph Delorme, Les Consolations, Pensées d'Août, Paris, Charpentier.

SCHULTENS Albert, $1748^{1}, 1767^{2}$, Thomae Erpenii Grammatica Arabica Cum Fabulis Locmanni. Accedunt Excerpta Anthologiae Veterum Arabiae Poetarum Quae Inscribitur Hamasa Abi Temmam, Lugduni Batavorum, Luchtmans.

SILVESTRE DE SACY Antoine-Isaac, 1806, Kitāb al-anīs al-mufìd li-l-țālib al-mustafìd. Chrestomathie arabe, ou extraits de divers écrivains arabes, tant en prose qu'en vers, à l'usage des élèves de l'École spéciale des Langues Orientales vivantes, par A. I. Silvestre de Sacy, Tome I ${ }^{\text {er }}$ contenant le texte arabe, Paris, Imprimerie Impériale.

SILVESTRE DE SACY Antoine-Isaac, 1816, Calila et Dimna ou Fables de Bidpaï, en arabe ; précédées d'un mémoire sur l'origine de ce livre, et sur les diverses traductions qui ont été faites dans l'Orient, et suivies de la Moallaka de Lébid, en arabe et en françois; par M. Silvestre de Sacy, Paris, Imprimerie Royale.

SILVESTRE DE SACY Antoine-Isaac, 1826-1827, Kitāb al-anīs al-mufìd li-l-țālib al-mustafìd wa-ǧāmi al-šudūrr min manzūm wa-mantūur. Chrestomathie arabe, ou extraits de divers écrivains arabes, tant en prose qu'en vers, avec une traduction et des notes à l'usage des Elèves de l'Ecole royale et spéciale des Langues Orientales vivantes, seconde édition corrigée et augmentée par M. le Baron Silvestre de Sacy, Paris, Imprimerie Royale, Tome I Ir et Tome II, 1826 ; Tome III, 1827.

WELLHAUSEN Julius, 1884, Skizzen und Vorarbeiten, Erstes Heft. 1. Abriss der Geschichte Israels und Juda's. 2. [Letzer Teil der] ${ }^{32}$ Lieder der Hudhailiten, Arabisch und Deutsch, Berlin, Georg Reimer.

32 Le titre complet n'apparaît qu'au début de la $2^{e}$ partie (p. 103). Au début de la partie arabe apparaît le titre ašă al al-Hudaliyyīn mā baqiya min al-nusha al-luġduniyya gayr mațbü' (" poésies des Huhaylites : ce qu'il reste du manuscrit de Leyde non imprimé »). 
